\title{
Clinical evaluation of temporomandibular joint disorder after orthognathic surgery in skeletal class II malocclusion patients
}

\author{
Jin-Hyun Jang, Sung-Keun Choi, Sung-Ho Park, Jin-Woo Kim, Sun-Jong Kim, Myung-Rae Kim \\ Department of Oral and Maxillofacial Surgery, Mokdong Hospital, \\ School of Medicine, Ewha Womans University, Seoul, Korea
}

\begin{abstract}
J Korean Assoc Oral Maxillofac Surg 2012;38:139-44)
Objectives: This study was performed in order to evaluate the occurrence of temporomandibular joint disorder after surgical correction of skeletal class II malocclusion.

Materials and Methods: This study included 21 patients who underwent orthognathic surgery for the correction of dentofacial deformities by a single surgeon at Mokdong Hospital, Ewha Womans University from 2000 to 2010. They underwent bilateral sagittal split ramus osteotomy for the treatment of undesirable mandibular advancement. The temporomandibular disorder (TMD) symptoms prior to surgery were recorded and the radiographic evaluation (panorama, bone scan, and magnetic resonance imaging [MRI]) of the post-surgery temporomandibular joint (TMJ) were assessed in order to evaluate condylar resorption, remodeling and disc displacement. The minimum follow-up period, including orthodontic treatment, was 12 months. Orthognathic procedures included 1 -jaw surgery ( $\mathrm{n}=8$ patients) and 2-jaw surgery ( $\mathrm{n}=13$ patients). The monocortical plate was used for bilateral sagittal split ramus osteotomy fixation.

Results: Among class II malocclusion patients with TMD symptom, clicking improved in 29.1\%, and maximum mouth opening increased from $34.5 \pm 2.1 \mathrm{~mm}$ to $37.2 \pm 3.5 \mathrm{~mm}$. The differences were not statistically significant, however. Radiographic changes in bone scan improved slightly based on the report by radiologist but not in TMJ dynamic MRI.

Conclusion: No particular improvements were found in patients with joint sound only. Patients with limitation of mouth opening showed an increase in the degree of opening, but the difference was not statistically significant $(P>0.05)$.
\end{abstract}

Key words: Orthognathic surgery, Class II malocclusion, Temporomandibular joint disorders, Clinical evaluation [paper submitted 2012. 3. 5 / revised 1st 2012. 4. 5, 2nd 2012. 5. 17 / accepted 2012. 5. 18]

\section{Introduction}

The clinical changes in temporomandibular disorder (TMD) before and after orthognathic surgery in Class II and Class III patients have been controversial. Wisth ${ }^{1}$ and Magnusson et al. ${ }^{2}$ reported the useful effect of orthognathic surgery on TMD patients based on the improvements of the limitation of mouth opening and joint sound found after surgery. According to O’Ryan and Epker ${ }^{3}$ and Sanders et al. ${ }^{4}$, however, orthognathic surgery actually had negative impact on the temporomandi-

\footnotetext{
Sun-Jong Kim

Department of Oral and Maxillofacial Surgery, School of Medicine, Ewha Womans University, 1071 Anyangcheon-ro, Yangcheon-gu, Seoul 158-710, Korea

TEL: +82-2-2650-5041 FAX: +82-2-2650-5764

E-mail:sjsj7777@ewha.ac.kr
}

(C) This is an open-access article distributed under the terms of the Creative Commons Attribution Non-Commercial License (http://creativecommons org/licenses/by-nc/3.0/), which permits unrestricted non-commercial use, distribution, and reproduction in any medium, provided the original work is properly cited. bular joint (TMJ). The temporomandibular symptoms may be developed after orthognathic surgery are caused by the change in fossa-condyle-disk structure and the adaptation of the neuromuscular system, and the change in fossa-condyledisk is directly caused by the lateral pterygoid when semirigid fixation is applied ${ }^{5}$. The direct change in condylar position can take place during surgical operation such as the application of rigid fixation. The improvement of clinical symptoms after orthognathic surgery can be explained by the occlusal stability after surgery and the reduction of emotional stress, whereas the occurrence of TMD after orthognathic surgery can be attributed to the condylar pressure due to inadequate fixation of proximal segment and change in the internal structure of the joint itself ${ }^{6}$.

The purpose of this study is to evaluate the change in the clinical symptoms of TMD postoperatively in Class II patients (based on Angle's classification of malocclusion) who received orthognathic surgery for mandibular advancement with Bilateral Sagittal Split Ramus Osteotomy (BSSRO). 


\section{Materials and Methods}

\section{Patients}

This study examined 21 patients ( 8 male and 13 female patients, average age: $23.3 \pm 4.2$ ) whose medical records and radiographs on TMD could be analyzed for a period of at least 1 year among the patients classified as Class II based on Angle's classification of malocclusion and who underwent orthognathic surgery mainly for malocclusion and deformity by the same surgeon at the Department of Oral and Maxillofacial Surgery in Mokdong Hospital from January 2000 to December 2010. All patients underwent BSSRO with semi-rigid fixation using titanium plates and screws (2-jaw surgery, $\mathrm{n}=13$, 1-jaw surgery, $\mathrm{n}=8$ ).

\section{Methods}

The 21 patients were divided into the following 4 groups according to the clinical symptoms of TMD: patients with joint sound without pain $(\mathrm{G} 1, \mathrm{n}=7)$; patients whose maximum mouth opening is $35 \mathrm{~mm}$ or less $(\mathrm{G} 2, \mathrm{n}=4)$; patients with TMJ pain with noise (G3, $n=5)$, and patients with limitation in mouth opening and pain $(\mathrm{G} 4, \mathrm{n}=5)$. The changes in symptoms were examined through clinical and radiological examination before the surgery, 2 months after the surgery, and 6 months after the surgery.(Table 1) Depending on the severity of the patient's symptom, bone scan or magnetic resonance imaging (MRI) was conducted additionally (bone scan, $\mathrm{n}=5$ and TMJ MRI, $\mathrm{n}=2$ ).

\section{Statistical analysis}

For a group consisting of G2 and G4 (maximum mouth opening $[\mathrm{MMO}]<35 \mathrm{~mm}$ ) with limitation in mouth opening, changes in MMO before and after the surgery were verified

Table 1. Classification of patients G4 PreOp and PostOp MMO

\begin{tabular}{ccccc}
\hline Patient & No. of patients & TMJ noise & LOM $(<35 \mathrm{~mm})$ & TMJ pain \\
\hline G1 & $\mathrm{n}=7$ & + & - & - \\
G2 & $\mathrm{n}=4$ & - & + & - \\
G3 & $\mathrm{n}=5$ & + & - & + \\
G4 & $\mathrm{n}=5$ & - & + & + \\
\hline
\end{tabular}

(PreOp: preoperative, PostOp: postoperative, MMO: maximum mouth opening, TMJ: temporomandibular joint, LOM: limitation of mouth opening, G1: group 1, G2: group 2, G3: group 3, G4: group 4)

Jin-Hyun Jang et al: Clinical evaluation of temporomandibular joint disorder after orthognathic surgery in skeletal class II malocclusion patients. J Korean Assoc Oral Maxillofac Surg 2012 in the paired t-test (SPSS version 12.0; SPSS Inc., Chicago, IL, USA). The degree of temporomandibular pain and sound before the surgery, 2 months, and 6 months after the surgery were compared, including the change in radiographic examination (chi-square test; SPSS Inc.).

\section{Results}

1. Clinical symptom changes after orthognathic surgery

1) Change in TMJ symptom of patient with joint sound (G1) Out of 7 patients who manifested joint sound (clicking, popping, and crepitus) in the clinical examination, 2 patients showed improvement in joint sound. Among patients who had joint sound only, 2 showed a limitation in mouth

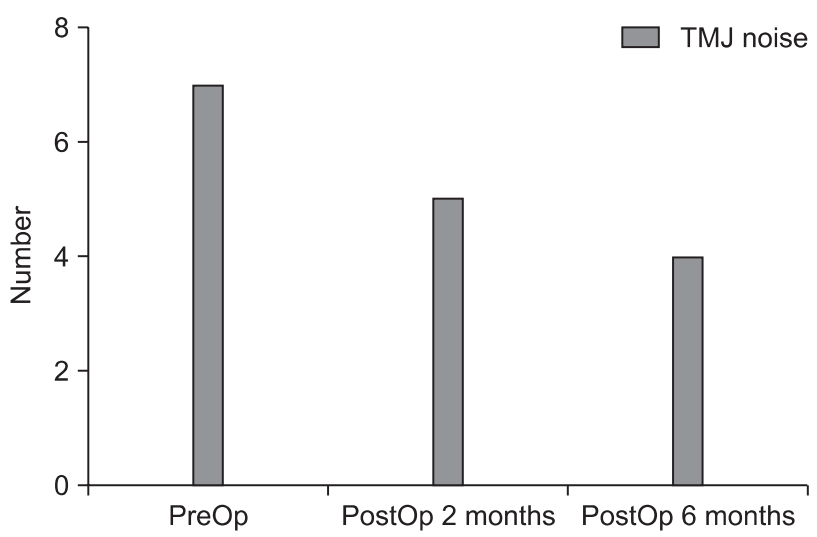

Fig. 1. Changes in TMJ noise after orthognathic surgery in group 1. (TMJ: temporomandibular joint, PreOp: preoperative, PostOp: postoperative)

Jin-Hyun Jang et al: Clinical evaluation of temporomandibular joint disorder after orthognathic surgery in skeletal class II malocclusion patients. J Korean Assoc Oral Maxillofac Surg 2012

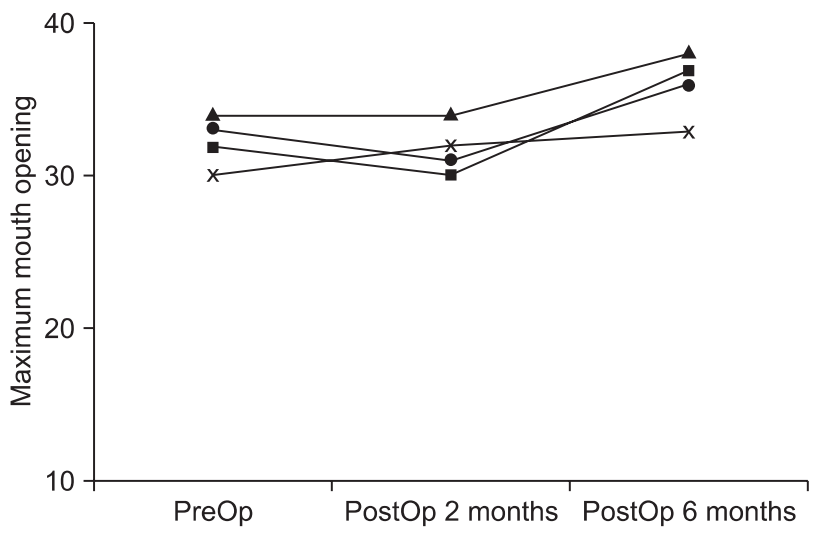

Fig. 2. Changes after operation in limitation of mouth opening only (group 2). (PreOp: preoperative, PostOp: postoperative) Jin-Hyun Jang et al: Clinical evaluation of temporomandibular joint disorder after orthognathic surgery in skeletal class II malocclusion patients. J Korean Assoc Oral Maxillofac Surg 2012 
opening 2 months after the surgery and 1 patient exhibited improvement 6 months after the surgery.(Fig. 1)

2) Change in temporomandibular symptom in patients with MMO lesser than $35 \mathrm{~mm}(\mathrm{G} 2)$

Out of 4 patients who manifested MMO lesser than $35 \mathrm{~mm}$ in the clinical examination, 1 showed increase in the mouth opening of up to $35 \mathrm{~mm}$ or more 6 months after the surgery; 1 patient did not manifested improvement. MMO increased by $2.3 \pm 1.2 \mathrm{~mm}(13.62 \%)$ on average, but the difference was not statistically significant $(P>0.05)$.(Fig. 2)

3) Change in temporomandibular symptom in patients with joint sound and pain (G3)

Out of 5 patients who had joint sound and pain in the clinical examination, 1 showed improvement for joint pain 6 months after the operation, and 3 patients, for joint sound.(Fig. 3)

4) Change in temporomandibular symptom in patients with limitation in mouth opening accompanied by joint pain (G4)

Out of 5 patients who showed a limitation in mouth

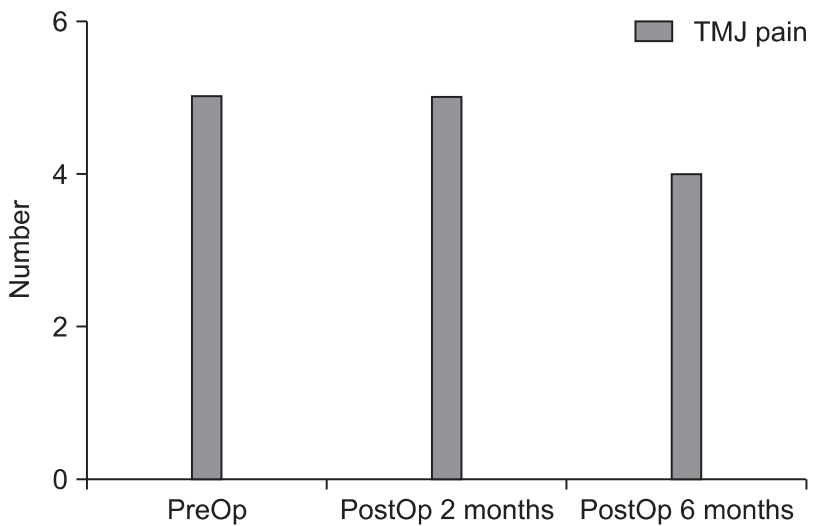

Fig. 3. Changes after the operation in group 3 patient (group 3). (TMJ: temporomandibular joint, PreOp: preoperative, PostOp: postoperative)

Jin-Hyun Jang et al: Clinical evaluation of temporomandibular joint disorder after orthognathic surgery in skeletal class II malocclusion patients. J Korean Assoc Oral Maxillofac Surg 2012 opening accompanied by joint pain in the clinical examination, 2 patients exhibited improvement on the limitation in mouth opening and joint pain 6 months after the surgery $(P<0.05)$.(Fig. 4, Tables 2, 3)

\section{Radiological evaluation (TMJ bone scan and MRI)}

Five patients of all groups examined in the nuclear medical test of TMJ, 1 manifested increase of uptake; other patients showed improvement or no change.(Table 4, Fig. 5)

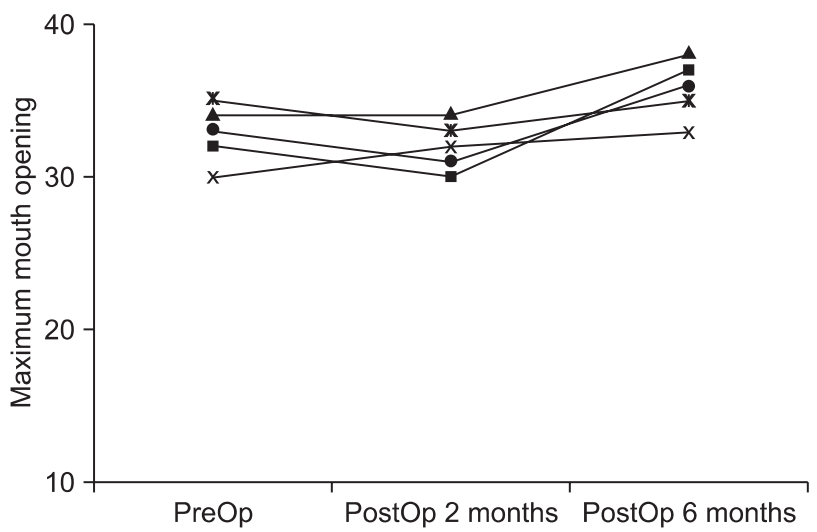

Fig. 4. Changes of mouth opening after the operation in limited mouth opening with TMJ pain (group 4). (TMJ: temporomandibular joint, PreOp: preoperative, PostOp: postoperative)

Jin-Hyun Jang et al: Clinical evaluation of temporomandibular joint disorder after orthognathic surgery in skeletal class II malocclusion patients. J Korean Assoc Oral Maxillofac Surg 2012

Table 2. PreOp and PostOp MMO of G4

\begin{tabular}{ccc}
\hline Patient & PreOp & PostOp 6 months \\
\hline Patient 1 & 32.00 & 35.00 \\
Patient 2 & 33.00 & 36.00 \\
Patient 3 & 34.00 & 40.00 \\
Patient 4 & 35.00 & 46.00 \\
Patient 5 & 32.00 & 45.00 \\
\hline
\end{tabular}

(PreOp: preoperative, PostOp: postoperative, MMO: maximum mouth opening, G4: group 4)

Jin-Hyun Jang et al: Clinical evaluation of temporomandibular joint disorder after orthognathic surgery in skeletal class II malocclusion patients. J Korean Assoc Oral Maxillofac Surg 2012

Table 3. Paired t-test of group 4

\begin{tabular}{|c|c|c|c|c|c|c|c|c|c|}
\hline \multicolumn{10}{|c|}{ Paired samples test ${ }^{1}$} \\
\hline & & \multicolumn{5}{|c|}{ Paired differences } & \multirow{3}{*}{$\mathrm{T}$} & \multirow{3}{*}{ df } & \multirow{3}{*}{ Sig. (2-tailed) } \\
\hline & & \multirow[t]{2}{*}{ Mean } & \multirow[t]{2}{*}{ Std. deviation } & \multirow[t]{2}{*}{ Std. error mean } & \multicolumn{2}{|c|}{$\begin{array}{l}95 \% \text { confidence interval } \\
\text { of the difference }\end{array}$} & & & \\
\hline & & & & & Lower & Upper & & & \\
\hline Pair 1 & PreOp-PostOp 6 months & -7.20000 & 4.60435 & 2.05913 & -12.91705 & -1.48295 & -3.497 & 4 & 0.025 \\
\hline
\end{tabular}

(Std.: standard, df: degree of freedom, Sig.: significant)

${ }^{1}$ No statistics are computed for one or more split files.

Jin-Hyun Jang et al: Clinical evaluation of temporomandibular joint disorder after orthognathic surgery in skeletal class II malocclusion patients. J Korean Assoc Oral Maxillofac Surg 2012 
Patients examined through MRI on the TMJ did not show any difference before and after the surgery.(Table 5, Figs. 6, 7).

\section{Discussion}

The purpose of this study is to obtain data for explaining

Table 4. Changes after the operation in bone scan findings

\begin{tabular}{cll}
\hline Patient & \multicolumn{1}{c}{$\begin{array}{c}\text { PreOp } \\
\text { TMJ bone scan findings }\end{array}$} & $\begin{array}{c}\text { PostOp (minimum 6 month after) } \\
\text { TMJ bone scan findings }\end{array}$ \\
\hline Patient 1 & Suggestive arthritis & Mild uptake \\
Patient 2 & Mild uptake & No activity on TMJ \\
Patient 3 & Increased uptake & Mild uptake \\
Patient 4 & Mild uptake & Increased uptake \\
Patient 5 & Mild uptake & Mild uptake \\
\hline
\end{tabular}

(PreOp: preoperative, PostOp: postoperative, TMJ: temporomandibular joint) Jin-Hyun Jang et al: Clinical evaluation of temporomandibular joint disorder after orthognathic surgery in skeletal class II malocclusion patients. J Korean Assoc Oral Maxillofac Surg 2012 the potential changes of TMD after the orthognathic surgery in $\mathrm{Cl}$ II patients. There are many types of temporomandibular symptoms, such as joint sound, limitation of mouth opening, pain. And the frequency of the symptoms also varies, ranging from $12-57 \%$ in preceding studies. In particular, the frequency of TMD was reported to be relatively high among patients with dentofacial deformities. According to Wisth ${ }^{1}$, TMD occurred in $71 \%$ of dentofacial deformity patients; White and Dolwick ${ }^{6}$ reported a $49.3 \%$ of occurrence, and Kerstens et al. ${ }^{7}$, a $16.2 \%$ of occurrence.

Kerstens et al. ${ }^{7}$, reported that $66 \%$ of the patients who had TMD before the surgery showed improvement after mandibular advancement, but that $11.6 \%$ exhibited receding of TMD symptom. Wolford et al. ${ }^{8}$, reported that $16 \%$ of 124 patients who had orthognathic surgery for mandibular advancement experienced temporomandibular pain, $64 \%$ of patients had joint sound, and $20 \%$ of patients showed both

Table 5. MRI changes in 2 patients

\begin{tabular}{cll}
\hline & PreOp MRI & \multicolumn{1}{c}{ PostOp MRI (minimum 6 month after) } \\
\hline Patient 1 & ADD without reduction & ADD without reduction, no change from PreOp MRI finding \\
Patient 2 & ADD without reduction & ADD without reduction, no change from PreOp MRI finding \\
\hline
\end{tabular}

(PreOp: preoperative, PostOp: postoperative, MRI: magnetic resonance imaging, ADD: anterior disc displacement)

Jin-Hyun Jang et al: Clinical evaluation of temporomandibular joint disorder after orthognathic surgery in skeletal class II malocclusion patients. J Korean Assoc Oral Maxillofac Surg 2012
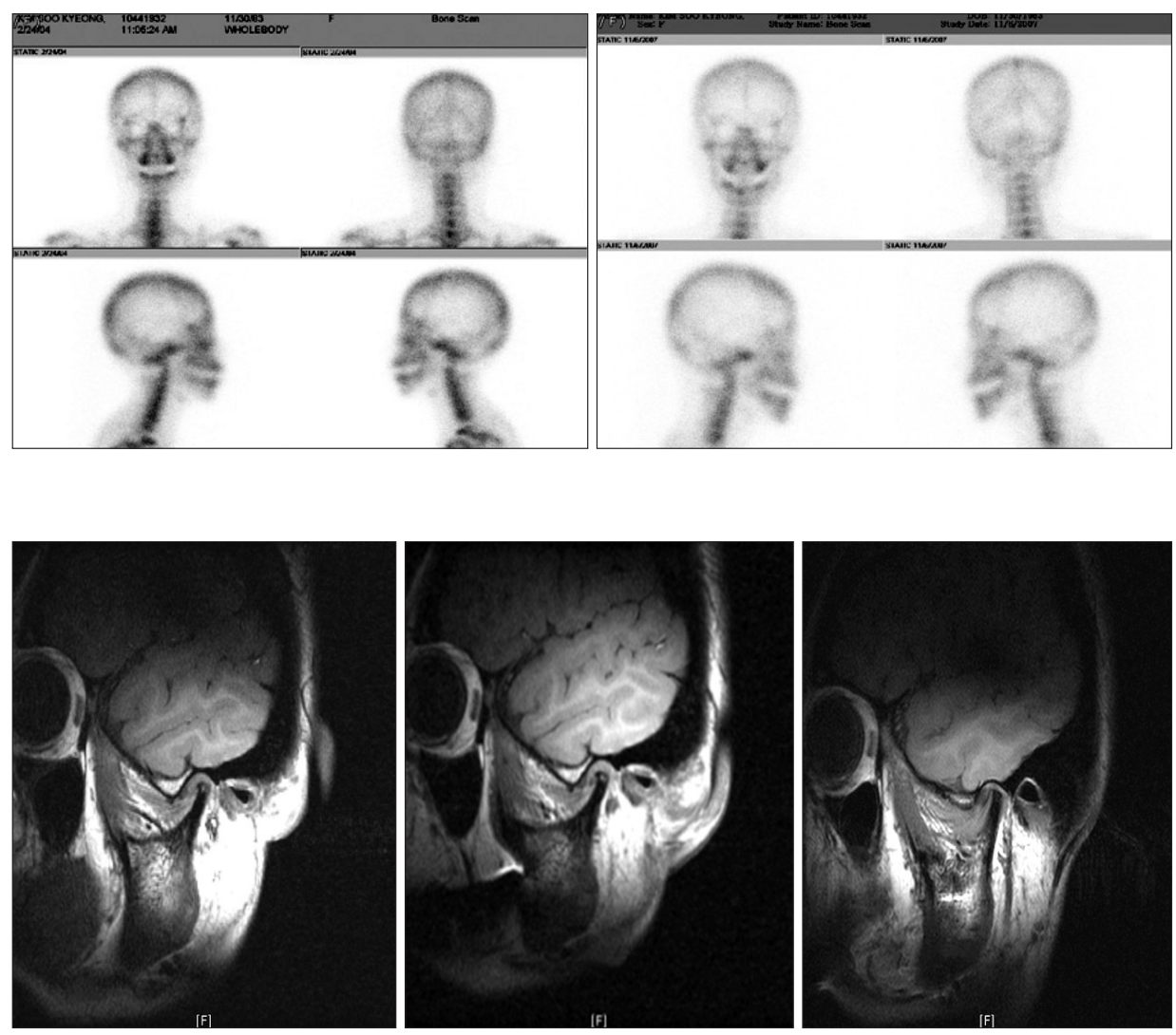

Fig. 6. Changes of magnetic resonance imaging after orthognathic surgery. 2 years, 1 month, and 1 year after orthognathic surgery. Anterior disc displacement has not changed (patient 1, 2-jaw). (LT: left)

Jin-Hyun Jang et al: Clinical evaluation of temporomandibular joint disorder after orthognathic surgery in skeletal class II malocclusion patients. J Korean Assoc Oral Maxillofac Surg 2012 

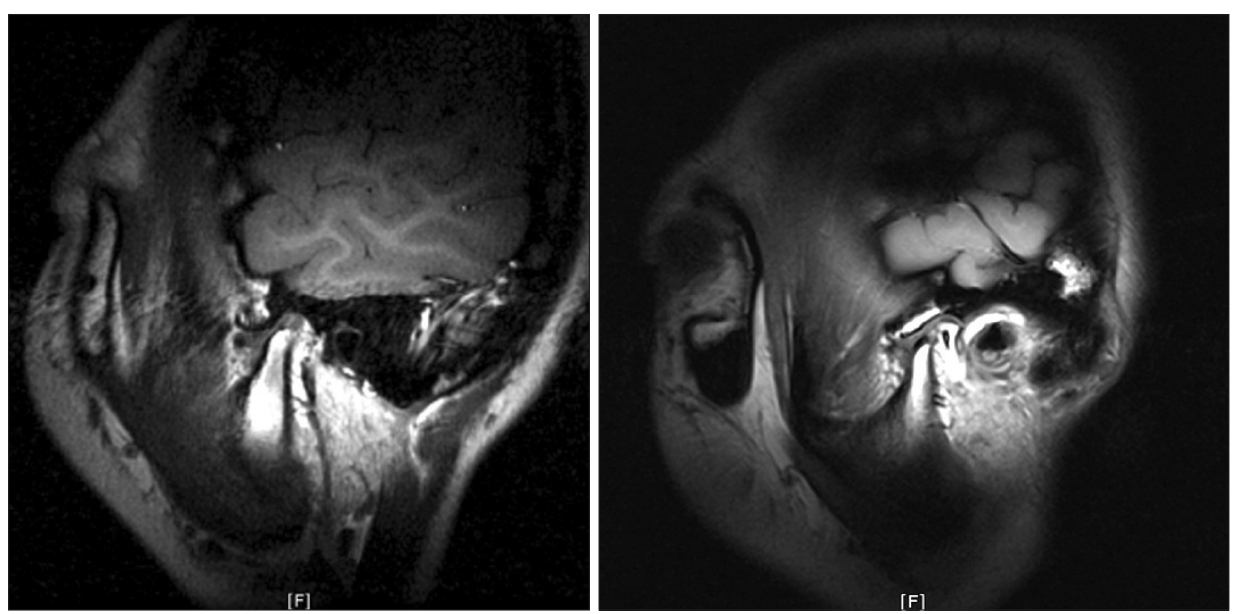

Fig. 7. Changes of magnetic resonance imaging after orthognathic surgery. 2 years before, and 1 year after orthognathic surgery. Anterior disc displacement has not changed (patient 2). (LT: left)

Jin-Hyun Jang et al: Clinical evaluation of temporomandibular joint disorder after orthognathic surgery in skeletal class II malocclusion patients. J Korean Assoc Oral Maxillofac Surg 2012

symptoms before the surgery; the symptoms worsened as the figures increased, with $24 \%$ of patients experiencing temporomandibular pain, $16 \%$ of patients having joint sound, and $60 \%$ manifesting both symptoms. As reported, there are great differences between doctors in their opinions on TMD symptoms after orthognathic surgery. Thus, predicting whether the symptoms would improve or worsen after the surgery in individual patients can be difficult. According to Proffit et al. ${ }^{9}$, TMD after orthognathic surgery occurs or worsens with the change in contact surface due to the movement of proximal segment; rigid or semi-rigid fixation after surgery can cause rotation or inclination of condylar axis, leading to condylar displacement that influences the condylar function. Panula et al. ${ }^{10}$ reported that orthognathic surgery can have functional improvement, but that TMD is not directly related to dentofacial deformity. Similar results were reported by Aoyama et al. ${ }^{11}$ and Farella et al. ${ }^{12}$.

In this study, the number of patients who had joint sound decreased from 20 before the surgery to 7 after the surgery; patients with pain during mouth opening decreased from 7 to 3 , and those have pain, from 5 to 2 . Considering all patients, 16 out of the 21 with TMD showed improvement in their symptoms. This can be interpreted as a result of the surgery, which stabilized occlusion and reduced the functional load to the TMJ.

Dervis and Tuncer ${ }^{13}$ studied 100 patients with TMD including 50 who had underwent orthognathic surgery and 50 who had not during a 2-year treatment period, and reported that surgical correction can have a positive impact on TMJ pain and dysfunction. Similarly, Pahkala and Kellokoski ${ }^{14}$ studied 82 patients with TMD and reported that BSSRO reduces TMD effectively. In this study, $62 \%$ of the patients who had at least one of the symptoms before the surgery exhibited improvement after the surgery.

According to the review of recent literatures on the relationship between TMD and orthognathic surgery by the American Association of Oral and Maxillofacial Surgeons (AAOMS), orthognathic surgery has a positive impact on TMD; nonetheless, the journals more or less lacked accuracy in methodology, deduction and objectivity ${ }^{15}$.

Precisely evaluating the impact of orthognathic surgery on TMD will require many studies and considerable understandings based on a large number of cases. For the further studies, there should be investigation with mathematical and statistical data through a number of studies and cases with regard to the trend of TMD in Class II patients in relation to the surgery.

\section{Conclusion}

This study is to investigate the effect of orthognathic surgery for Class II malocclusion patients on improvement in TMD. The change of symptoms on TMD was examined through clinical and radiological examinations, and the following conclusions were as follows.

Firstly, no particular improvements were found in patients with joint sound only.

Secondly, patients with limitation of mouth opening showed an increase in the degree of opening, but the difference was not statistically significant $(P>0.05)$.

Thirdly, patients with joint sound and pain showed improvement on pain, but the difference was not statistically significant $(P>0.05)$.

Finally, patients with limitation of mouth opening and pain showed improvement on the limitation of mouth opening and pain both $(P<0.05)$. 


\section{References}

1. Wisth PJ. Mandibular function and dysfunction in patients with mandibular prognathism. Am J Orthod 1984;85:193-8.

2. Magnusson T, Ahlborg G, Svartz K. Function of the masticatory system in 20 patients with mandibular hypo- or hyperplasia after correction by a sagittal split osteotomy. Int J Oral Maxillofac Surg 1990;19:289-93.

3. O'Ryan F, Epker BN. Surgical orthodontics and the temporomandibular joint. I. Superior repositioning of the maxilla. Am J Orthod 1983;83:408-17.

4. Sanders B, Kaminishi R, Buoncristiani R, Davis C. Arthroscopic surgery for treatment of temporomandibular joint hypomobility after mandibular sagittal osteotomy. Oral Surg Oral Med Oral Pathol 1990;69:539-41.

5. Lee JY, Kim SG, Seung JH, Ahn JM. Alterations of temporomandibular joint symptom after orthognathic surgery. J Korean Assoc Maxillofac Plast Reconstr Surg 2003;25:448-51.

6. White CS, Dolwick MF. Prevalence and variance of temporomandibular dysfunction in orthognathic surgery patients. Int J Adult Orthodon Orthognath Surg 1992;7:7-14.

7. Kerstens HC, Tuinzing DB, van der Kwast WA. Temporomandibular joint symptoms in orthognathic surgery. J Craniomaxillofac Surg 1989;17:215-8.

8. Wolford LM, Reiche-Fischel O, Mehra P. Changes in temporomandibular joint dysfunction after orthognathic surgery. J Oral Maxillofac Surg 2003;61:655-60.
9. Proffit WR, Phillips C, Dann C 4th, Turvey TA. Stability after surgical-orthodontic correction of skeletal Class III malocclusion. I. Mandibular setback. Int J Adult Orthodon Orthognath Surg 1991;6:7-18.

10. Panula K, Somppi M, Finne K, Oikarinen K. Effects of orthognathic surgery on temporomandibular joint dysfunction. A controlled prospective 4-year follow-up study. Int J Oral Maxillofac Surg 2000;29:183-7.

11. Aoyama S, Kino K, Kobayashi J, Yoshimasu H, Amagasa T. Clinical evaluation of the temporomandibular joint following orthognathic surgery--multiple logistic regression analysis. J Med Dent Sci 2005;52:109-14.

12. Farella M, Michelotti A, Bocchino T, Cimino R, Laino A, Steenks MH. Effects of orthognathic surgery for class III malocclusion on signs and symptoms of temporomandibular disorders and on pressure pain thresholds of the jaw muscles. Int J Oral Maxillofac Surg 2007;36:583-7.

13. Dervis E, Tuncer E. Long-term evaluations of temporomandibular disorders in patients undergoing orthognathic surgery compared with a control group. Oral Surg Oral Med Oral Pathol Oral Radiol Endod 2002;94:554-60.

14. Pahkala RH, Kellokoski JK. Surgical-orthodontic treatment and patients' functional and psychosocial well-being. Am J Orthod Dentofacial Orthop 2007;132:158-64.

15. Lindenmeyer A, Sutcliffe P, Eghtessad M, Goulden R, Speculand B, Harris M. Oral and maxillofacial surgery and chronic painful temporomandibular disorders--a systematic review. J Oral Maxillofac Surg 2010;68:2755-64. 